\title{
Full high-throughput sequencing analysis of differences in expression profiles of long noncoding RNAs and their mechanisms of action in systemic lupus erythematosus
}

Hui Ye ${ }^{1 *+}$, Xue Wang ${ }^{1+}$, Lei Wang ${ }^{2 \dagger}$, Xiaoying Chu ${ }^{1+}$, Xuanxuan Hu${ }^{1}$, Li Sun ${ }^{3}$, Minghua Jiang ${ }^{4}$, Hong Wang ${ }^{4}$, Zihan Wang ${ }^{5}$, Han Zhao ${ }^{2}$, Xinyu Yang ${ }^{6^{*}}$ and Jianguang Wang ${ }^{1 *}$

\begin{abstract}
Background: The specific function of long noncoding RNAs (InCRNAs) in systemic lupus erythematosus (SLE) and the mechanism of their involvement in related pathological changes remain to be elucidated, so, in this study, we analyzed the differences in the expression profiles of IncRNAs and their mechanisms of action in SLE using full high-throughput sequencing, bioinformatics, etc. methods.

Methods: We used high-throughput sequencing to detect differences in the expression profiles of IncRNAs, miRNAs, and mRNAs in PBMCs from patients with SLE at the genome-wide level. Next, we predicted target genes of 30 lincRNAs (long intergenic noncoding RNAs) by constructing a coexpression network of differential lincRNAs and mRNAs and identified the role of lincRNAs. Then, we analyzed the coexpression network of 23 optimized lincRNAs and their corresponding 353 miRNAs, evaluated the cis- and trans-effects of these lincRNAs, and performed GO and KEGG analyses of target genes. We also selected 8 lincRNAs and 2 newly discovered IncRNAs for q-PCR validation and IncRNA-miRNA-mRNA analysis. Finally, we also analyzed respectively the relation between IncRNAs and gender bias in SLE patients using RT-qPCR, the relation between Systemic Lupus Erythematosus Disease Activity Index score and the "IFN signature" using ELISA, and the relation between the differential expression of IncRNAs and a change in the number of a cell type of PBMCs in SLE patients using RT-qPCR.

Results: The profiles of 1087 IncRNAs, 102 miRNAs, and 4101 mRNAs in PBMCs significantly differed between patients with SLE and healthy controls. The coexpression network analysis showed that the network contained 23 lincRNAs and 353 mRNAs. The evaluation of the cis- and trans-effects showed that the 23 lincRNAs acted on 704 target genes. GO and KEGG analyses of the target genes predicted the biological functions of the 23 lincRNAs. qPCR validation showed 7 lincRNAs and 2 novel IncRNAs were identical to the sequencing results. The ceRNA network contained 7 validated lincRNAs, 15 miRNAs, and 155 mRNAs. In addition, the differential expression of InCRNAs may be gender dependent in SLE patients, SLE patients also exhibit a robust "IFN signature," and PBMCS exhibiting differential expression of IncRNAs may be due to a change in the number of a cell type.

(Continued on next page)
\end{abstract}

\footnotetext{
* Correspondence: wmcyh@wmu.edu.cn; cyrillyoung@163.com;

wz_wjg@163.com

${ }^{\dagger}$ Hui Ye, Xue Wang, Lei Wang and Xiaoying Chu contributed equally to this work.

${ }^{1}$ Department of Biochemistry, School of Basic Medical Sciences, Wenzhou

Medical University, Wenzhou 325035, Zhejiang, China

${ }^{6}$ Department of Medicinal Chemistry, School of Pharmaceutical Sciences,

Wenzhou Medical University, Wenzhou 325035, Zhejiang, China

Full list of author information is available at the end of the article
}

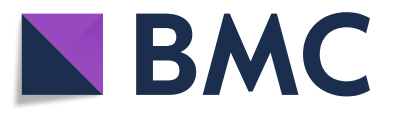

(c) The Author(s). 2019 Open Access This article is distributed under the terms of the Creative Commons Attribution 4.0 International License (http://creativecommons.org/licenses/by/4.0/), which permits unrestricted use, distribution, and

reproduction in any medium, provided you give appropriate credit to the original author(s) and the source, provide a link to the Creative Commons license, and indicate if changes were made. The Creative Commons Public Domain Dedication waiver (http://creativecommons.org/publicdomain/zero/1.0/) applies to the data made available in this article, unless otherwise stated. 
(Continued from previous page)

Conclusion: This work determined specific IncRNAs that play important biological functions in the pathogenesis of lupus and provided a new direction for diagnosis and treatment of disease.

Keywords: High-throughput sequencing analysis, Expression profiles, Long noncoding RNA, Mechanisms, Systemic lupus erythematosus

\section{Introduction}

Systemic lupus erythematosus (SLE) is a chronic autoimmune disease with complicated clinical manifestations and leads to multiple systemic impairments. The course of the disease repeats, and aggravation and remission alternate; however, the early symptoms are often atypical [1]. The main features of SLE are the presence of numerous pathogenic autoantibodies, complement depletion, and chronic inflammation. SLE has a clear gender tendency; that is, it mainly affects women of childbearing age (a ratio of 1:9 for men to women). According to statistics, 150 cases of SLE occur among 100,000 people. Among people suffering from SLE for 5 years, those with kidney failure or died account for 15\% [2]. The development of SLE is jointly influenced by various factors, such as genes, environment, epigenetics, and hormones. However, the precise pathogenesis of SLE remains unclear.

Long noncoding RNAs (lncRNAs) are endogenous transcript RNA molecules that have a length of more than 200 nucleotides [3] and have no protein-encoding capacity. LncRNAs were previously ignored and considered as nonfunctional mRNA transcription by-product. LncRNA was first discovered in 2002 during a large-scale, genome-wide sequencing of the rat cDNA library [4]. Since then, many studies have explored lncRNAs and reported their involvement in regulating the expression of protein-coding genes and epigenetic genes through various mechanisms, such as epigenetic modification, alternative splicing, and post-transcriptional and translational control [5]. LncRNAs are categorized into five groups [3]: (1) long intergenic noncoding RNAs (lincRNA) between genes, (2) antisense lncRNAs, (3) intronic transcript lncRNAs, (4) promoter-associated lncRNAs, and (5) UTR-associated IncRNAs. In the present work, the most studied and functional lincRNAs were analyzed. About $20 \%$ of lincRNAs in cells influenced gene expression through epigenetic effects by binding to and targeting chromatin-modifying complexes, such as PRC2 [6]. LncRNAs are thought to be associated with cell differentiation and activation and play an important regulatory role in the differentiation and activation of immune cells in congenital and acquired immune systems [7]. Therefore, lncRNAs could be related to various autoimmune diseases, such as rheumatoid arthritis, psoriasis, and sicca syndrome [8]. Scholars in the field of transcriptome research have focused on determining lncRNAs that are associated with the pathogenesis of SLE. In this disease, differences in the expression of lncRNAs were found. $\mathrm{Wu}$ et al. reported the significant downregulation of linc0949 and linc0597 in peripheral blood mononuclear cells (PBMCs) of patients with SLE; the results also indicated that linc0949 expression was negatively correlated with the degree of disease activity and organ damage but positively correlated with complement C3 level [9]. However, the specific mechanism of action of IncRNAs in the development of SLE has not been elucidated yet. Current research on lncRNAs in SLE has relied on the results of ChIP analysis $[9,10]$ and did not perform high-throughput sequencing.

Comprehensively understanding the epigenetic and molecular mechanisms of SLE is the key to achieve early diagnosis, recommend appropriate treatment, and obtain good prognosis. This study uses full high-throughput sequencing technology to analyze the differences in lncRNA expression profiles between patients with SLE and controls (healthy individuals) at the whole-genome level. Specific lincRNA target genes were predicted, and lncRNAmiRNA-mRNA ceRNA networks were constructed. In addition, we also make experiments to confirm the following: whether differential expression of IncRNAs in our screen was gender dependent, whether SLE patients exhibited a robust or weak "IFN signature," and whether PBMCs from SLE patients (as compared with healthy donors) exhibited differential expression of lncRNAs due to a change in the number of a cell type (e.g., lymphocytes, monocytes, and DCs) in peripheral blood. Results would be used as the basis for the study of specific lncRNAs involved in the specific pathogenesis of SLE.

\section{Methods}

\section{Participants and sample collection}

This study recruited 147 individuals with SLE (aged $41.3 \pm 10.6$ years) who were outpatients or inpatients of the Rheumatology and Immunology Division of the First Affiliated Hospital of Wenzhou Medical University and the Second Affiliated Hospital of Wenzhou Medical University from March 2016 to October 2018. The selected cases were consistent with the SLE diagnostic criteria revised by the American Rheumatism Association in 1997. Blood specimen collection and Systemic Lupus Erythematosus Disease Activity Index (SLEDAI) [11] statistical 
analysis were conducted before administering glucocorticoids and immunosuppressive agents to the patients. The normal control group consisted of 117 healthy donors (aged $40.8 \pm 12.1$ years) with no history of autoimmune diseases or immunosuppressive therapy. The control group matched the patient group in terms of age and gender. This study was approved by the Research Ethics Committee of Wenzhou Medical University (No. 2018925) and obtained informed consents from all participants. In high-throughput sequencing, 30 patients with SLE and 30 healthy donors were divided into 10 groups (among them, patients were grouped according to their SLEDAI scores: group 1 consisted of individuals with no activity (3.2 \pm 0.9 points), group $2(6.3 \pm 0.7$ points) and group 3 ( $8.3 \pm 0.5$ points) consisted of those with mild activity, group 4 consisted of those with moderate activity (13.0 \pm 1.2 points), and group 5 consisted of those with severe activity (19.0 \pm 2.6 points); each group was mixed and sequenced with 6 samples of the same SLEDAI score). In the study of a relation between lncRNAs and gender bias in SLE patients, 37 healthy donors, 19 SLE patients (containing male and female), 26 female SLE patients, and 13 male SLE patients were used for RT-qPCR. In the study of a relation between SLEDAI score and the "IFN signature," 33 SLE patients (12 inactive (SLEDAI score $<4$ ) and 21 active (SLEDAI score >4)) and 20 healthy donors were used for enzyme-linked immunosorbent assay (ELISA). In the study of a relation between the differential expression of lncRNAs and a change in the number of a cell type of PBMCs in SLE patients, 26 SLE patients and 30 healthy donors were used for the isolation of PBMCs, T cells, B cells, monocytes, DCs, and RT-qPCR.

\section{High-throughput sequencing analysis}

High-throughput sequencing was completed by RiboBio Co. Ltd. (Guangzhou, China). The operation procedure was briefly described as follows: After total RNA extraction, the Ribo-ZeroTM rRNA Removal Kit (Epicenter, Madison, WI, USA) was used to remove rRNA and achieve RNA fragmentation (average fragment length of approximately $200 \mathrm{nt}$ ). Single-stranded cDNA was synthesized by reverse transcription, and then double-stranded cDNA was synthesized. After purification of double-stranded cDNA, the terminal repair was performed. The sample was added with primers, amplified, and purified through PCR for library construction according to RNA species. After the quality inspection of the library, ten sets of samples (five vs. five) were sequenced using the Illumina HiSeqTM 2500 sequencing platform (Illumina, San Diego, CA, USA). Bioinformatics analysis of raw sequence data was conducted, and the ensemble transcript database was used to annotate the results.
Real-time quantitative reverse transcriptase polymerase chain reaction validation

The expressions of selected lncRNAs were screened and verified by qRT-PCR. Glyceraldehyde 3-phosphate dehydrogenase (GAPDH) mRNA was used as an internal control. The primers are listed in Additional file 1: Table S1. For reverse transcription (RT) reactions, SuperScript First-Strand Synthesis System (Invitrogen, Carlsbad, CA, USA) was used. In brief, random primers were used and reacted at $42{ }^{\circ} \mathrm{C}$ for $60 \mathrm{~min}$ and at $72^{\circ} \mathrm{C}$ for $10 \mathrm{~min}$ to obtain a cDNA template. SYBR Green PCR Master Mix (Applied Biosystems, Foster City, CA, USA) was used for PCR amplification. The reaction system was added with $10 \mu \mathrm{l} 2 \times$ SYBR Green mix, $6.8 \mu \mathrm{l}$ of cDNA, $0.8 \mu \mathrm{l}$ upstream primer $(5 \mu \mathrm{M}), 0.8 \mu \mathrm{l}$ downstream primer $(5 \mu \mathrm{M})$, and $1.6 \mu \mathrm{l}$ of RNase-free $\mathrm{H}_{2} \mathrm{O}$ up to the total volume of $20 \mu \mathrm{l}$. All experiments were performed in triplicates. The final qPCR data of lncRNA were processed using $2 \Delta \Delta$ ct method against GAPDH for normalization.

\section{Prediction of 30 screened lincRNA target gene}

The function of 30 screened lincRNA was predicted through bioinformatics analysis to determine its effect on target genes. Previous works indicated that the lincRNA gene could regulate post-transcriptional steps. The role of lincRNAs and their target genes was predicted by establishing the relationship between differential lincRNAs and differential mRNAs. The roles of lincRNAs and their target genes included cis- and trans-actions [12]. The lincRNA cis-action is predicted by searching all coding genes within the 10-kb upstream and downstream of the target lincRNA, and these neighboring genes may be regulated by lincRNAs. The lincRNA trans-action is predicted based on nucleic acid base pairing.

\section{Gene Ontology and Kyoto Encyclopedia of Genes and Genomes analysis}

Gene Ontology (GO) analysis annotates differentially expressed genes in terms of cell composition, molecular function, and biological processes [13]. Kyoto Encyclopedia of Genes and Genomes (KEGG) analysis is an effective method for predicting the potential biological function of differentially expressed genes [14]. KEGG analysis is also used to provide differentially expressed mRNAs with annotation information for signal transduction and disease pathways and background knowledge of gene pathways and functional studies. In the GO and KEGG analyses, $P$ value $<0.05$ was used as the screening standard.

\section{The correlation between IFN- $\alpha$ and disease activity in SLE patients}

The venous blood of 33 SLE patients (12 in the no-activity group (SLEDAI score $<4$ ) and 21 in the activity group (SLEDAI score $>4$ )) and 20 healthy donors 
(see the "Participants and sample collection" section for details) was collected aseptically ( $5 \mathrm{ml}$ per person) and left undisturbed for $2 \mathrm{~h}$ at room temperature. After serum separation, it was centrifuged $(500 \mathrm{~g} \times 10 \mathrm{~min})$ to aspirate the supernatant serum, which was dispensed into an Eppendorf tube and stored in a refrigerator at $-20^{\circ} \mathrm{C}$. According to the manufacturer's instructions, an interferon (IFN)- $\alpha$ ELISA Kit (purchased from PBL Biomedical Laboratories, USA) was used to detect the IFN- $\alpha$ level in SLE patients and normal control group. The operation procedure was briefly described as follows: The diluted coated antibody was added to $100 \mu \mathrm{l}$ pores of ELISA plate and stored for $48 \mathrm{~h}$ at $4{ }^{\circ} \mathrm{C}$. The ELISA plate was washed three times with washing solution, and then $200 \mu \mathrm{l}$ of blocking buffer per well was added for $1 \mathrm{~h}$ at $37^{\circ} \mathrm{C}$. the ELISA plate was washed three times, and the sample to be tested was added with the negative control; the double-diluted enzyme-labeled antibodies were added, $100 \mu \mathrm{l}$ per well; they were placed at $37^{\circ} \mathrm{C}$ for $1 \mathrm{~h}$; the ELISA plate was washed three times; the prepared ABTS color-substrate solution was added, $100 \mu \mathrm{l}$ per well; at $37^{\circ} \mathrm{C}$, the color was developed for $30 \mathrm{~min}$ by water bath; the optical density (OD) values at $410 \mathrm{~nm}$ were measured with an ELISA reader; a standard curve was drawn; the corresponding results of each serum sample were determined, and then Prism Software was used to make a scatter plot and a correlation analysis graph.

\section{Isolation of PBMCs, T cells, B cells, monocytes, DCs, and RT-qPCR of IncRNA NR_034053.2}

The whole blood of 26 SLE patients and 30 healthy donors (see the "Participants and sample collection" section for details) was collected aseptically ( $30 \mathrm{ml}$ per person) in EDTA collection tubes, and PBMCs were isolated by density-gradient centrifugation with Ficoll-Paque Premium (GE Healthcate), according to the instructions. For the subsets of PBMCs isolation, the fresh PBMCs were incubated for $15 \mathrm{~min}$ at $4{ }^{\circ} \mathrm{C}$ with fluorescent-conjugated monoclonal antibodies: anti-CD3-PerCP-Cy5.5, anti CD14-PE, and anti-CD19-APC (all from BD Biosciences). Stained cells were sorted on a BD FACSAria III (BD Biosciences). $\mathrm{T}$ cells were identified as $\mathrm{CD}^{+} / \mathrm{CD} 19^{-}$. B cells were collected if cells were $\mathrm{CD} 19^{+} / \mathrm{CD}^{-}$. Monocytes were isolated if cells were $\mathrm{CD} 14^{+} / \mathrm{CD}^{-}$. And then, monocyte cells were then cultured for 5-7 days in RPMI 1640 supplemented with $1000 \mathrm{U} / \mathrm{ml}$ granulocyte/macrophage colony-stimulating factor (GM-CSF) and $1000 \mathrm{U} / \mathrm{ml}$ interleukin (IL)-4 (PeproTech, Rocky Hill). For monocyte-derived dendritic cell (moDC) maturation, $1 \mu \mathrm{g} / \mathrm{ml}$ lipopolysaccharide (LPS; Escherichia coli type 055:B6; Sigma) was added to the medium at day 6.

Then, the total RNAs of PBMC, T cells, monocytes, B cells, and DCs of the above SLE patients were extracted respectively, and the total RNAs from PBMC and DCs of healthy donors were extracted; next, RT-qPCR for lncRNA NR_034053.2 of the above cells was performed respectively, and internal reference was GAPDH (note: for the primer sequence, please see Additional file 1: Table S1).

\section{Construction of LncRNA-mRNA coexpression network} An lncRNA-mRNA coexpression network was constructed based on the specific expression levels of mRNAs and the normalized signal intensity of lncRNA to determine the interaction between differentially expressed mRNAs and differentially expressed lncRNAs. After obtaining the expression values of all genes, their correlation with one another and $P$ value were calculated and screened $(C O R>0.85)$. A given node would be filtered out if the Pearson correlation coefficient between any pair of nodes is greater than COR. Only COR value was used for screening.

\section{LncRNA-miRNA-mRNA network analysis}

The expression profiles of differentially expressed lncRNAs, miRNAs, and mRNAs and the prediction feature of bioinformatics software were used to construct lncRNA-miRNA-mRNA networks. miRanda, PITA, and RNAhybrid were used to predict the recognition regions of lncRNAs and miRNAs. Further screening was conducted, and the prediction results of each software program were collated. Two or more software predictions were used as candidate reciprocal miRNAs for lncRNAs. For candidate miRNAs, miRDB, miRTarBase, miRWalk, and TargetScan software were used. The results of the three software programs and the common prediction results were regarded as miRNAs corresponding to mRNAs. The lncRNAs and their candidate miRNAs and corresponding mRNAs were selected.

\section{Statistical analysis}

Continuous variables are expressed as means (SD). The Student $t$ test or one-way analysis of variance was used to compare continuous variables. All $P$ values were estimated in a two-tailed fashion. Differences were considered to be statistically significant at $P<0.05$. Data were analyzed using SPSS 22.0 (SPSS Inc., IL, USA). The relationships between the expression levels of IFN- $\alpha$ and Systemic Lupus Erythematosus Disease Activity Index (SLEDAI) were analyzed by Pearson's correlation coefficient. Mapping was completed using GraphPad Prism 6. To highlight the differences in the expression during sequencing, we used Audics for correcting the obtained $P$ value to the $q$ value; the lower the $q$ value is, the more significant the difference in gene expression will be. 


\section{Results}

Expression profiles of differentially expressed IncRNAs, miRNAs, and mRNAs

Second-generation sequencing technology was used to detect lncRNAs in PBMCs in the five groups of patients with SLE and the five groups of healthy controls. The application of volcano plots was used to directly show the overall distribution of lncRNAs and mRNAs in the two groups, and scatter maps were used to demonstrate the distribution of miRNAs (Fig. 1a-c). Using $\mid \log 2$ (fold_change) $\mid 1$, $q<0.001$ as the standard, we determined significant expression differences in 1087 lncRNAs (141 significant upregulations and 946 significant downregulations). Among the 47 lncRNAs that differed more than fivefold in number, 25 were upregulated and 22 were downregulated. ENST00000437947.1 upregulation is the most obvious (fold change up, 38.11160073), and NR_006881.1 downregulation is the most obvious (fold change down, 66.77770783). Of these differentially expressed lncRNAs, 184 belong to lincRNAs (33 were significantly upregulated and 151 were significantly downregulated), and 220 belong to antisense lncRNAs (37 were significantly upregulated and 183 were significantly downregulated).

In recent years, the role of lincRNAs has been described in several diseases and has become another star molecule following microRNA. After comprehensive analysis of the expression volume (RPKM), differential multiples, and lincRNA-derived genes, we screened 30 lincRNAs (15 upregulated and 15 downregulated) located near the SLE susceptibility locus for further study. The selected lincRNAs are shown in Additional file 2: Table S2 and Additional file 3: Table S3. We used a clustering heat map (Fig. 2a) to show the differences in the expression levels of the 30 lincRNAs between SLE and the control groups. We found 102 differential miRNAs (39 significantly upregulated and 63 significantly downregulated) and 4101 differential mRNAs (770 significantly upregulated and 3331 significantly downregulated). The discovery of these transcript differences laid the foundation for exploring the pathogenesis of lupus at the molecular level.
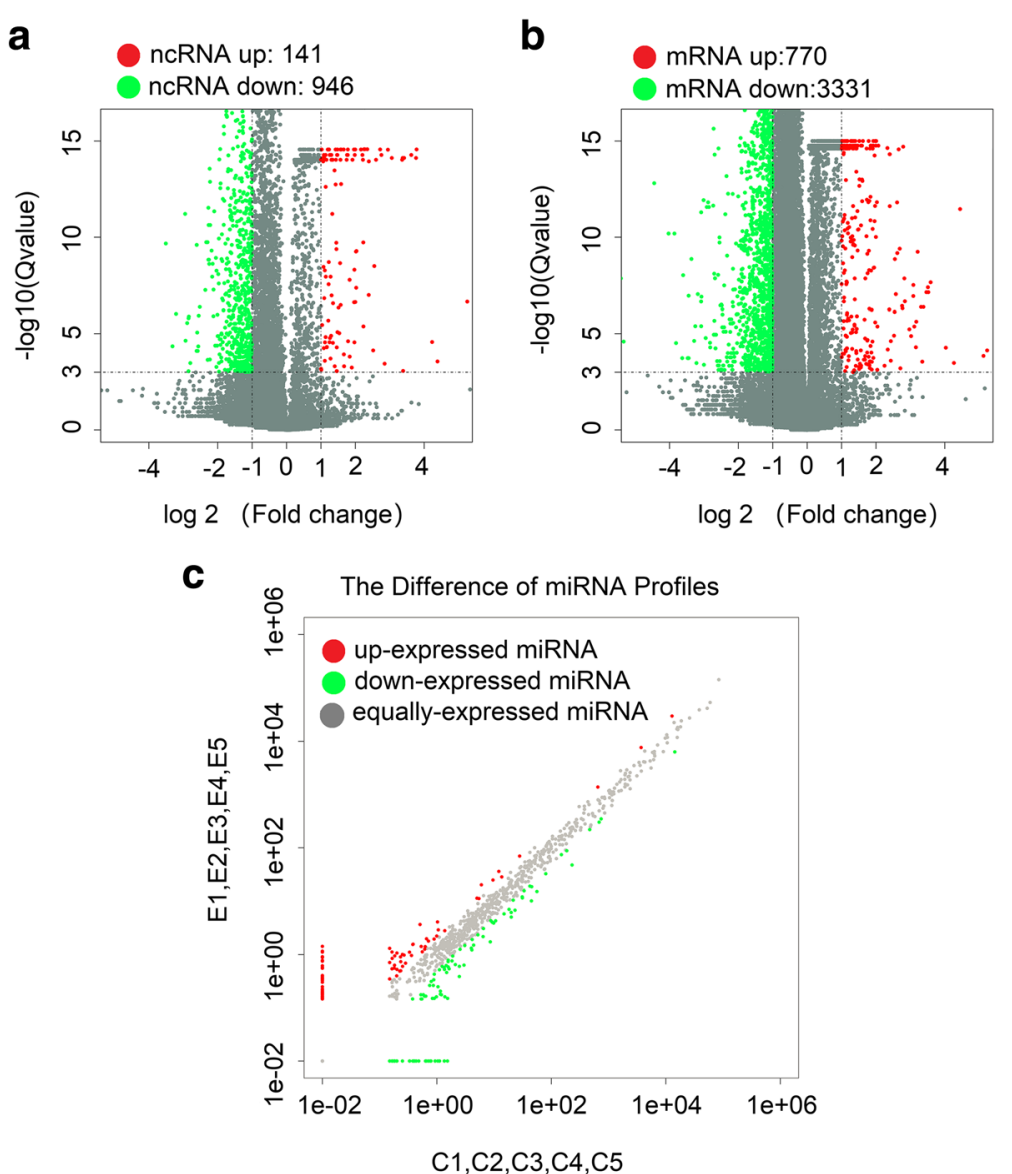

Fig. 1 High-throughput sequencing to reveal differences in the expression profiles of IncRNAs, mRNAs, and miRNAs between the SLE and control groups. a Differentially expressed IncRNA volcano maps between the SLE and NC groups. b Differentially expressed mRNA volcano plots between the SLE and NC groups. c Differentially expressed miRNA scatter maps between the SLE and NC groups 


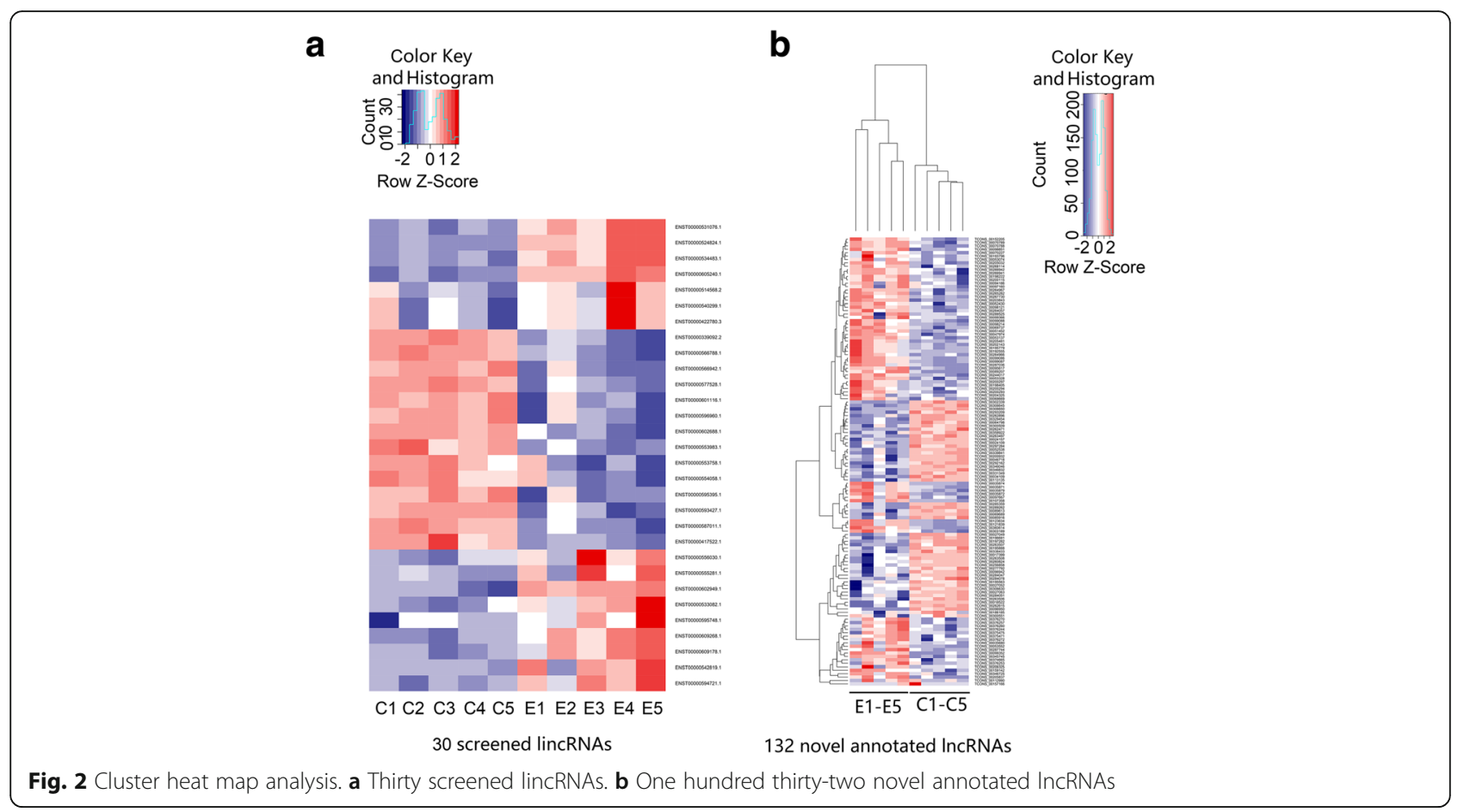

\section{Identification and differential expression analysis of novel IncRNAs}

A large number of novel promising lncRNAs in SLE have yet to be further screened because of the limitation of using microarray technology, which could only focus on known candidate lncRNAs preset on the chip. High-throughput sequencing not only can detect the expression of known lncRNAs but also can discover novel lncRNAs. In this study, we identified novel lncRNAs through the following stringent filter steps: (1) filter genes and lncRNAs with known filter database, (2) identify RNAs longer than $200 \mathrm{nt}$, (3) determine RNAs with predicted open reading frames $(\mathrm{ORF})<300 \mathrm{nt}$, (4) filter RNA with protein domain (Pfam), and (5) filter RNA with coding potential. We screened transcripts with lncRNA characteristics in new transcripts step-by-step and defined a novel lncRNA with a coding potential score of less than -1 in Coding Potential Calculator (CPC) software. We found a total of 5029 novel lncRNAs; among which, 132 showed significant differences and included 77 with significantly high expression and 55 with significantly low expression (| $\log 2$ (fold_change) $\mid>1, q<0.001)$. A clustering heat map was drawn to show the significant differences (Fig. 2b). Fifty novel lncRNAs had a difference multiple of 5 times and included 26 upregulated and 24 downregulated. The expression of TCONS_00205837 increased the most (fold change up, 67.72275292), whereas that of TCONS 00157166 decreased the most (fold change down, 51.89499278). We selected novel lncRNAs located near the SLE susceptibility locus for q-PCR validation. TCONS_00195779 was located at 2p25.2, where Linc00487 was also located. Linc00487 was confirmed to be related to B cell development. TCONS_00027049 and IL-10 were located at 1q31.3. IL-10 was found to be involved in immunoregulatory disorders of SLE [15]. Compared with the annotated lncRNAs, novel lncRNAs exhibited higher expression level and more significant differences in the sequencing results. Hence, these novel lncRNAs must be further investigated.

\section{lincRNAs and two novel IncRNAs showed expression profiles consistent with the sequencing results}

To screen lncRNAs with specific functions and verify the sequencing results, we specifically screened 8 lincRNAs (4 upregulated: ENST00000524824.1, ENST00000531076.1, ENST00000534483.1, and ENST00000542819.1; 4 downregulated: ENST00000596960.1, ENST00000566788.1, ENST00000601116.1, and ENST00000577528.1) and 2 novel lncRNAs (1 upregulated: TCONS00195779_1; 1 downregulated: TCONS00027049_1) for qRT-PCR validation. Samples were obtained from PBMCs of 30 patients with SLE and 30 healthy controls. These lncRNAs have relatively high expression levels, with large difference multiples, and are all located near the susceptibility genes. In the 4 upregulated lincRNAs, 3 lincRNAs were verified successfully, and their difference from ENST000 00542819.1 was not statistically significant (Fig. 3a). The ENST00000524824.1 expression increased with the largest multiples (up to 4.49 times). The expression level of 
a

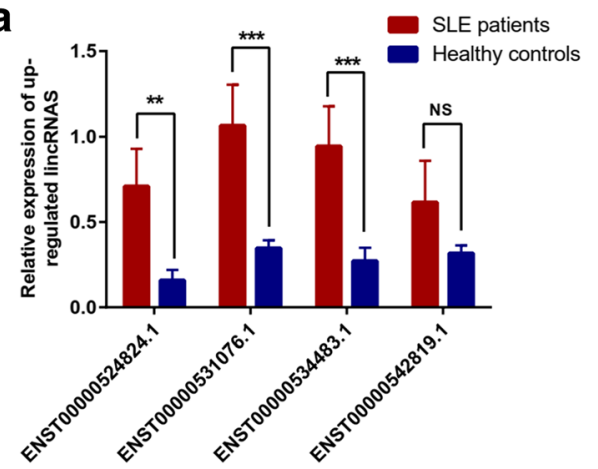

C

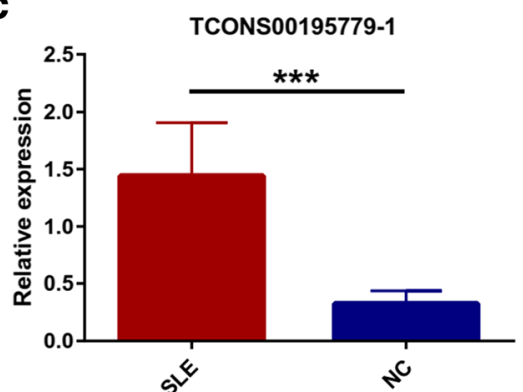

b

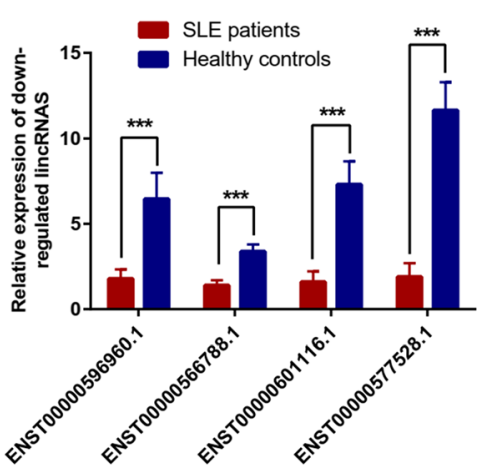

d

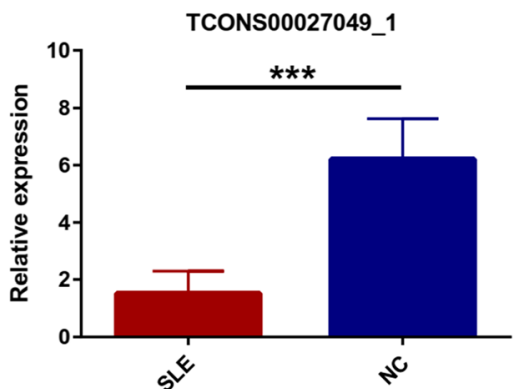

Fig. 3 The qRT-PCR validation of 8 selected lincRNAs and 2 novel IncRNAs between 30 SLE patients and healthy controls. a Relative expression of 4 upregulated lincRNAs. b Relative expression of 4 downregulated lincRNAs. c The relative expression of upregulated novel IncRNA TCONS00195779_1. $\mathbf{d}$ The relative expression of upregulated novel IncRNA TCONS00027049_1

ENST00000531076.1 increased 3.07 times and that of ENST00000534483.1 increased 3.47 times. All 4 downregulated lincRNAs were verified successfully. The ENST00000577528.1 expression decreased the most significantly (up to 6.14 times), followed by ENST0000 0601116.1 (4.59 times) and ENST00000596960.1 (3.61 times), and finally ENST00000566788.1 (2.42 times, Fig. 3b). In addition, differences in the expression of newly discovered lncRNAs were verified by q-PCR (Fig. 3c, d). The expression of TCONS00195779_1 increased 4.39 times and that of TCONS00027049_1 decreased 4.06 times. Seven lincRNAs and 2 novel lncRNAs showed expression profiles consistent with the sequencing results. This finding provides a basis for further analysis of the specific mechanism of action of lncRNAs.

lincRNAs may act on 704 target genes, forming a total of 890 IncRNA-gene connections

Many studies reported that lncRNAs can act by regulating the transcription of nearby coding genes [16, 17]. LncRNAs can also regulate the expression of genes at the post-transcriptional level by forming doublestranded complexes with mRNA [18]. In the present study, we established the relationship between differential lincRNAs and differential mRNA by predicting lincRNA action mode (cis- or trans-action). We identified 30 target genes that may act as lincRNAs. The prediction results indicate that 23 of 30 lincRNAs may act on 704 target genes, forming a total of 890 lincRNA-gene connections (Additional file 4: Figure S1). The seven remaining lincRNAs had no potential target genes; of which, ENST00000601116.1 has the most target genes (270); ENST00000534483.1, ENST000 00524824.1, and 1ENST00000531076.1, which are from ENSG00000255328.1, have 134 target genes. ENST0 0000566788.1 from ENSG00000260539.1 also has 134 target genes. ENST00000577528.1 may have effects on 60 target genes.

\section{GO and pathway analyses of 23 selected lincRNAs on 740 differential target genes}

To further clarify the biological roles of the 23 selected lincRNAs in SLE, we performed GO and pathway analyses on 740 differential target genes (Fig. 4). The analysis of the KEGG signaling pathway showed that the functional changes in 27 pathways had statistical significant difference between the SLE and the control groups $(P<0.05)$. These pathways included 118 abnormally expressed genes. The pathogenesis of lupus was found to be reacted to the following: metabolic pathways (the highest enrichment, $P=2 \times 10^{-6}$ ), p53 signaling pathway (second 


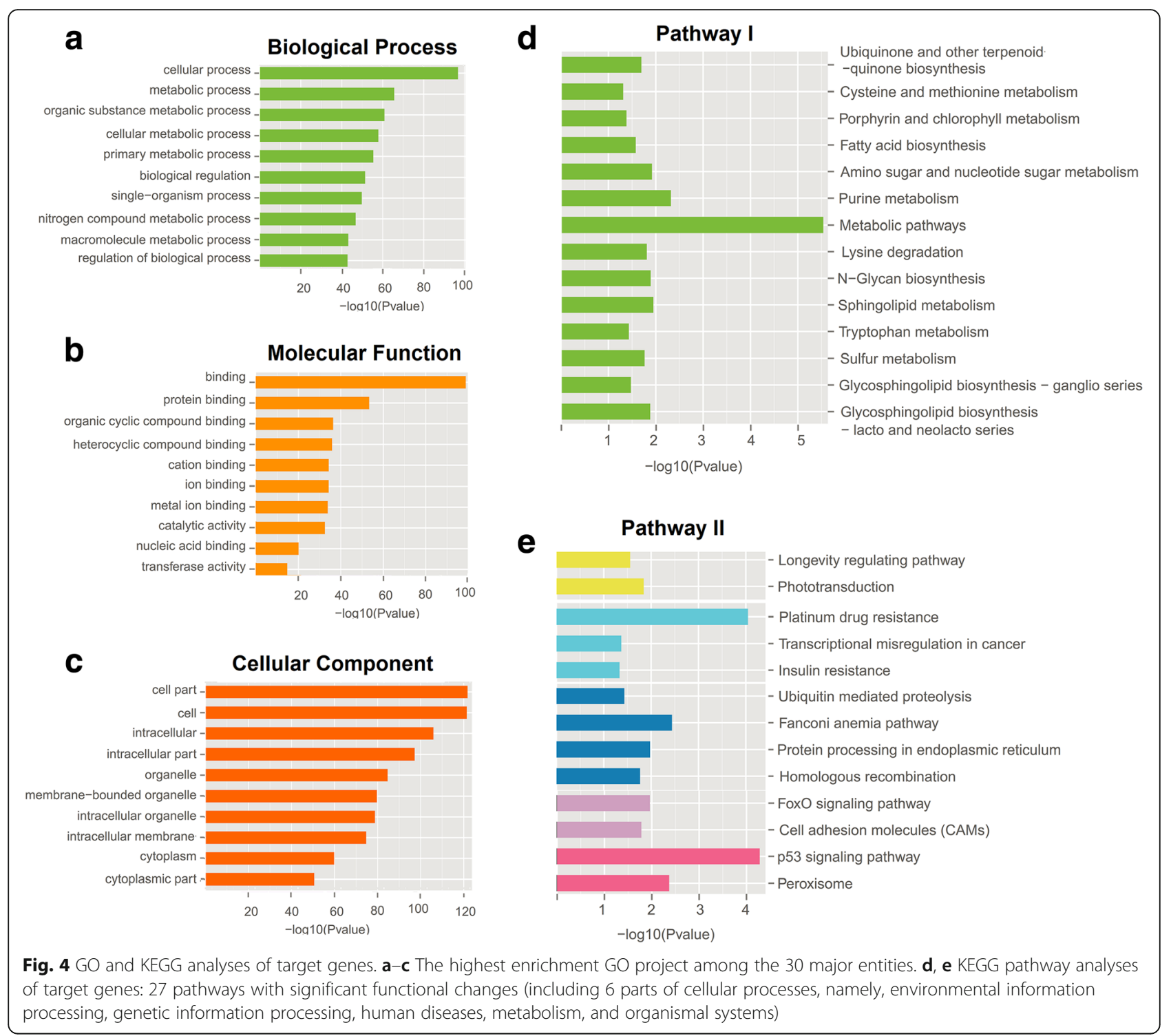

highest enrichment, $\left.P=5.25 \times 10^{-5}\right)$, and other pathways involved in FoxO signaling $(P=0.01)$, sphingolipid metabolism $(P=0.011)$, and cell adhesion molecules (CAMs) $(P=0.016)$ [19-21]. The GO project enrichment of the target genes included 1175 biological processes, 235 molecular functions, and 207 cellular components $(P<0.05)$, which mainly involves metabolism, cell signal transduction, DNAI binding and transcription, cell cycle, cytoskeleton, cell adhesion, apoptosis, and antigen presentation. The enrichment of cellular processes $(P=1.62 \times$ $\left.10^{-97}\right)$, metabolic processes $\left(P=2.24 \times 10^{-66}\right)$, binding $\left(P=1.58 \times 10^{-99}\right)$, and other GO stems was found to be the highest. Hence, lincRNAs can participate in the pathogenesis of lupus by affecting these functions.

\section{The differential expression of IncRNAs may be gender} dependent in SLE patients

SLE in patients exhibits a strong gender bias, so we wondered whether differential expression of lncRNAs in our screen was gender dependent. There is an increased incidence and prevalence of systemic lupus erythematosus in females, which might involve $\mathrm{X}$ chromosome inactivation [22]. TSIX is a long noncoding RNA, which protects the active- $\mathrm{X}$ from ectopic silencing once $\mathrm{X}$-inactivation has commenced [23]. As shown in Fig. 5a, the expression level of TSIX in SLE patients was significantly higher than that in the Nc group (healthy donors), and the difference was significant $(P<0.0001)$. The expression level of TSIX in female SLE patients was also significantly higher than that in male SLE patients $(P=$ 0.003) (Fig. 5b). These results indicated that in SLE 
a

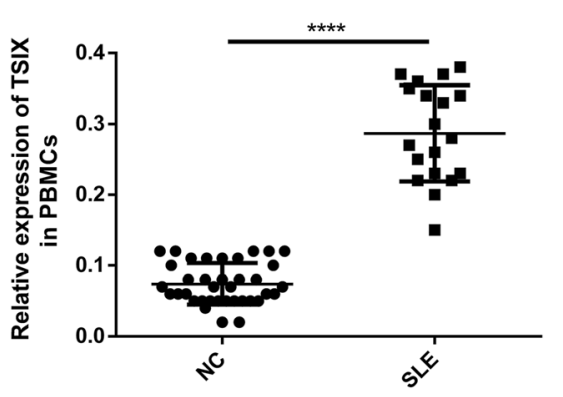

C

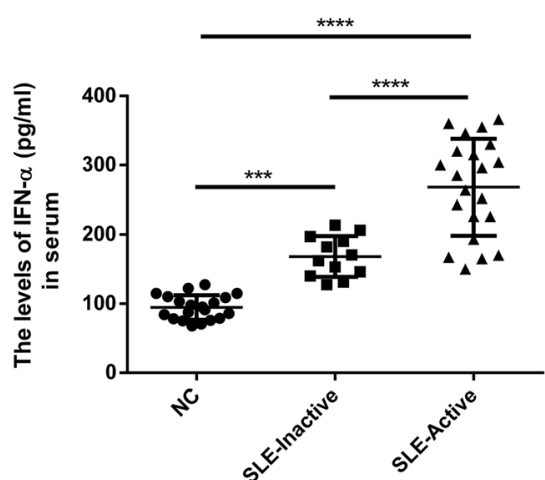

b

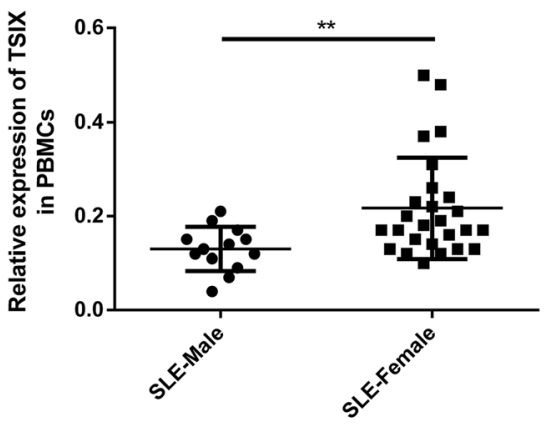

d

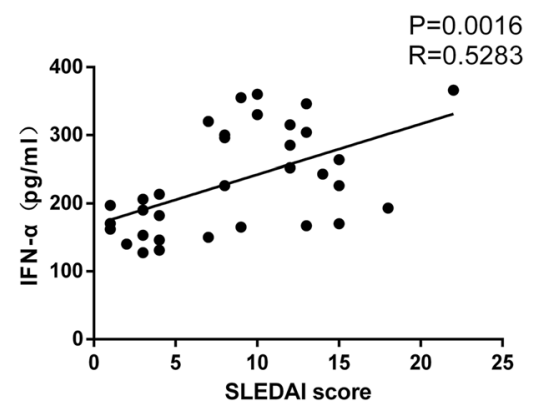

Fig. 5 SLE patients exhibit a strong gender bias and a robust "IFN signature." a TSIX expressions in PBMCs of 19 patients with SLE and 37 healthy donors (NC) were analyzed by qPCR. b TSIX expressions in PBMCs of 13 male SLE patients and 26 female SLE patients were analyzed by qPCR. ${ }^{* *} P<0.01,{ }^{* * *} P<0.001,{ }^{* * *} P<0.0001$. c IFN-a expressions in the serum of 12 patients with inactive SLE, 21 patients with active SLE, and 20 healthy donors (NC) were analyzed by ELISA. ${ }^{* *} P<0.001,{ }^{* * * *} P<0.0001$. d Nonparametric correlation (Spearman) was performed to assess the correlation between IFN- $a$ and SLEDAI score in patients with SLE $(n=33)$

patients, the upregulation of TSIX may promote the inactivation of the $\mathrm{X}$ chromosome by protecting active- $\mathrm{X}$ from ectopic silence.

\section{A positive correlation between IFN- $\alpha$ and disease activity in SLE patients}

Because interferon (IFN)- $\alpha$ is an important regulator of the body's inflammation and immune response, we wondered whether SLE patients exhibited a robust or weak "IFN signature" and further whether a particular SLEDAI score associated with the "IFN signature" or not. As shown in Fig. 5c, the IFN- $\alpha$ level in the serum of SLE patients is significantly higher than that in the normal control group (healthy donors) with significant difference $(P<0.001)$. The IFN- $\alpha$ level in the serum of SLE patients in the active group is also significantly higher than that in the non-active group $(P<0.0001)$. And there was a positive correlation between IFN- $\alpha$ expression and SLEDAI score in patients with SLE $(R=0.5283, P=$ 0.0016) (Fig. 5d). These results indicate that in SLE patients, the overexpressed IFN- $\alpha$ level correlates positively with the disease activity.
PBMCs exhibiting differential expression of IncRNAs may be due to a change in the number of a cell type

DCs from SLE patients produce much higher levels of the IFN- $\alpha$. PBMCs from SLE patients (as compared with healthy donors) exhibited differential expression of IncRNAs due to a change in the number of a cell type (e.g., lymphocytes, monocytes, and DCs) in the peripheral blood. So, we performed tests with lncRNA NR_034053.2 as an example; the level of lncRNA NR_034053.2 expression was determined in PBMCs obtained from SLE patients and healthy controls by qRT-PCR analysis. As shown in Fig. 6a, increased lncRNA NR_034053.2 expression was detected in the PBMCs from SLE patients compared with that from healthy donors $(P<0.0001)$. To determine the cellular specificity of lncRNA NR_034053.2 expression, we examined the expression of lncRNA NR_034053.2 in the main subsets of PBMC ( $T$ cells, monocytes, and B cells) from SLE patients. LncRNA NR_034053.2 was expressed at substantially increased levels in monocytes compared with $\mathrm{T}$ and $\mathrm{B}$ cells $(P<0.0001)$ (Fig. 6b). In addition, DCs from PBMC of SLE patients showed notably enriched lncRNA NR_034053.2 expression as compared 


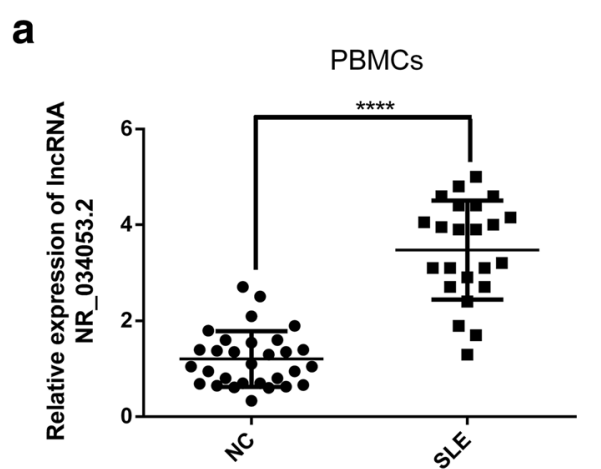

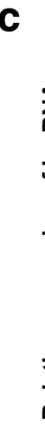

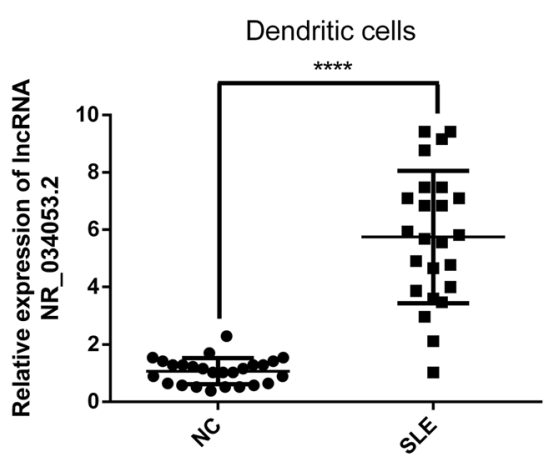

b

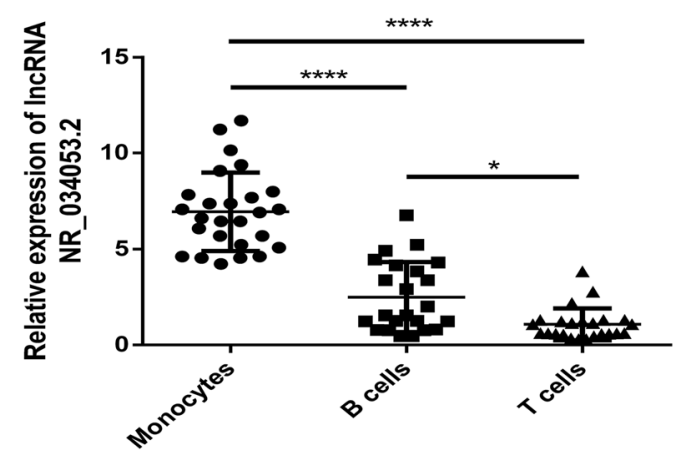

Fig. 6 The relative expression of InCRNA NR_034053.2 was determined by qPCR in SLE patients and healthy donors (NC). a Expression of IncRNA NR_034053.2 in PBMCs of SLE patients and healthy donors (NC) was determined by qPCR analysis. $\mathbf{b}$ Expression of IncRNA NR_034053.2 in monocytes, B cells, and T cells from SLE patients. c IncRNA NR_034053.2 expression was upregulated in DCs of SLE patients compared with healthy donors. ${ }^{*} P<0.05,{ }^{* * *} P<0.0001$

with healthy controls $(P<0.0001)$ (Fig. 6c), implying that the DCs are the primary lncRNA NR_034053.2-expressing cell type.

\section{LincRNA-mRNA expression pairs were built using 23 lincRNAs and 353 mRNAs}

Thirty differentially expressed lincRNAs and 4101 differentially expressed mRNAs were analyzed through a construction of a coexpression network. The network was built using 23 lincRNAs and 353 mRNAs, forming 3784 IncRNA-mRNA expression pairs; of which, 2652 had a positive correlation, 1130 had a negative correlation, and 1 mRNA can bind to 1-17 lincRNAs. One lincRNA can bind to 16-284 mRNAs. For example, ENST0000 0339092.2 could be expressed with 284 upregulated mRNAs. We found that 62 coexpressed mRNAs were involved in KEGG pathway changes. As shown in Additional file 5: Figure S2, Additional file 6: Figure S3, and Additional file 7: Figure S4, the lincRNA-mRNA coexpression network participates in pathways related to lupus (e.g., 23 differential lincRNAs and 23 differential mRNAs participate in metabolic pathways). In the p53 signaling pathway, 3 mRNAs interact with 17 lincRNAs. In the FoxO signaling pathway, 11 lincRNAs can bind to
3 mRNAs and, in addition, can be combined with the lncRNA target gene prediction above.

The ceRNA network was predicted using 9 validated IncRNAs, 15 miRNAs, and 155 mRNAs

Through bioinformatics software prediction, we constructed regulatory networks among lncRNAs, miRNAs, and mRNAs by using 7 validated lincRNAs and 2 novel lncRNAs. miRanda, PITA, and RNAhybrid were used to predict differentially expressed miRNAs that may bind to lncRNAs. Two or more software consensus predictions were used as candidate miRNAs. We identified 9 differential lncRNAs and 41 differential miRNAs that formed 54 lncRNA-miRNAs (Additional file 8: Figure S5). To narrow the scope and facilitate functional research, we selected 40 miRNAs based on the expression level and sequenced the multiple of the 102 differential miRNAs. Several miRNAs (such as miR-31-5p [24]) have been studied in SLE (cluster analysis, Additional file 9: Figure S6). By comparing the 40 miRNAs with the candidate miRNAs, we identified 7 differential lncRNAs and 15 differential miRNAs, which formed 19 lncRNA-miRNA pairs. We then used miRDB, miRTarBase, miRWalk, and TargetScan 
software to predict differentially miRNAs that can bind to miRNAs. We also used 3 or more software programs to jointly predict the results of the target gene. The ceRNA network contained 7 validated lncRNAs (3 upregulated and 4 downregulated), 15 miRNAs (3 upregulated and 12 downregulated), and 155 mRNAs (57 upregulated and 98 downregulated), with a total of 304 pairs of lncRNA-miRNA-mRNA predicted. The numbers of pairs between the 3 upregulated lncRNAs (ENST00000524824.1, ENST00000534483.1, TCONS_ 00195779) and the downregulated miRNAs were 1, 2, and 10, respectively, corresponding to 6 and 10 downregulated mRNAs and 64 upregulated mRNAs (Fig. 7). The numbers of pairs between the 4 downregulated lncRNAs (ENST00000566788.1, ENST00000577528.1, ENST00000596960.1, and TCONS_00027049) with the upregulated miRNAs were $2,1,1$, and 2 respectively corresponding to 98, 3, 25, and 98 downregulated mRNAs (Additional file 10: Figure S7). LncRNAs, miRNAs, and mRNAs can form a regulatory network through interaction and restricting 1 another in the gene network to participate in the physiological and pathological processes of SLE.

\section{Discussion}

SLE is a common autoimmune disease that is characterized by excessive activation of $\mathrm{T}$ and $\mathrm{B}$ lymphocytes and increased production of autoantibodies and involves many systems or organs (such as the skin, kidney, blood, nervous system). Although the use of immunosuppressive agents and new therapeutic interventions has increased the survival rate of patients with SLE over the past 10 years, some patients died due to severe disease activity in the internal organs [25]. Relapse and individualized treatment are key issues in controlling lupus. However, the key aspects of immune dysfunction and the pathogenesis of SLE remain unclear.

Abnormalities in epigenetic mechanisms play an important role in the occurrence and development of SLE. In the past, noncoding transcripts were considered to be "noise" in transcription due to their lack of or low coding capacity [26]. However, subsequent studies indicated that $98 \%$ of ncRNAs in DNA transcripts play an important role in epigenetic modifications, particularly in gene regulation and maintaining physiological processes, such as cell and tissue homeostasis. The most well-known ncRNAs are microRNAs (miRNAs), which are 21-23 nt

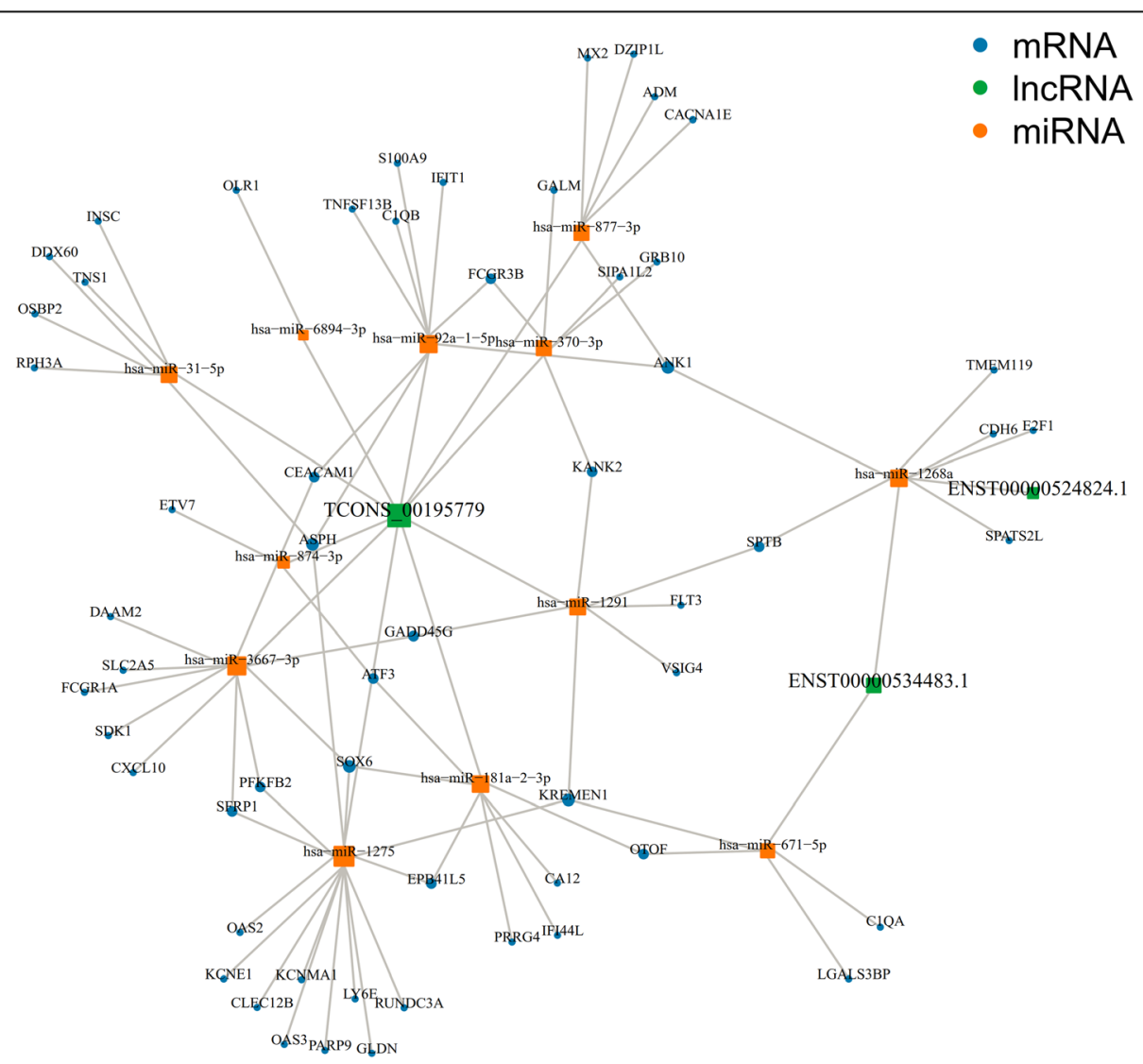

Fig. 7 The mapping network of IncRNA-miRNA-mRNA interactions between 3 upregulated IncRNAs, 12 downregulated miRNAs, and 57 upregulated mRNAs 
long and have been identified as an important regulator of SLE-related signaling pathways and genes [27], such as miR-146a, miR-155 [28], and miR-142-3p/5p [29]. Moreover, lncRNAs, which account for $80 \%$ of ncRNAs, are considered the star molecules following microRNAs and could influence the occurrence and development of diseases at the gene, transcription, and protein levels. LncRNAs exhibit specific temporal and spatial expression, and most of them show fine subcellular localization. LncRNAs are widely expressed in macrophages, monocytes, dendritic cells (DCs), neutrophils, $\mathrm{T}$ cells, and B cells and are closely related to their development, differentiation, and activation. In recent years, the roles of lncRNAs in autoimmune diseases have been widely reported. Manama et al. found that the expression levels of Gas5 in peripheral blood B cells and CD4+ $T$ cells were significantly downregulated in patients with SLE; as such, Gas 5 can inhibit the proliferation of T cells [30, 31]. LncRNA NEAT1 is mainly expressed in human monocytes; the expression of lncRNA NEAT1 is significantly upregulated in patients with SLE and positively correlated with the severity of the disease. As such, lncRNA NEAT1 can mediate inflammatory responses through the MAPK pathway [32]. LncRNA MEG3 was found to be involved in the pathogenesis of OA by regulating vascular endothelial growth factor [33]. LncRNA HOTAIR was considered a biomarker for the diagnosis of RA; this molecule was highly expressed in PBMCs and serum exosomes of patients with RA and promoted the extensive migration of activated macrophages [34]. Aberrant lncRNA expression, single-nucleotide polymorphisms, or base mutations at the corresponding positions are related to the occurrence and progression of autoimmune diseases.

Although few studies have been reported on lncRNAs in the past 20 years, the development of chips and high-throughput sequencing technologies has made it possible to identify lncRNAs on a large scale at the whole-genome level. Scholars have used chip technology to screen differential lncRNAs in SLE; the results promoted an in-depth study of the involvement of lncRNAs in the pathogenesis of lupus. However, the research results are inconsistent, and the expression levels obtained are contradictory. As a high-throughput transcriptome detection method based on second-generation sequencing, RNA-Seq has become the most promising technique because it can detect and quantify IncRNA expression without bias. The advantages of this technique include its ability to provide a highly dynamic range of expression and its superiority to chip analysis in terms of sensitivity and accuracy; Moreover, this technique does not need to predict the genomic locus of the transcribed region. At present, high-throughput sequencing of lncRNAs has been conducted in studies of tumors, such as colorectal [35] and liver cancers [36]; however, IncRNA sequencing of SLE has not been reported yet. Therefore, in this study, we use second-generation full-transcriptome high-throughput sequencing to detect differences in the expression levels of lncRNAs, miRNAs, and mRNAs in patients with lupus and healthy people. These three molecules were combined to analyze the interaction mechanism between lncRNAs and miRNAs as well as their interaction with mRNAs in SLE.

The sequencing results showed significant differences in the expression levels of 1087 lncRNAs (141 upregulated and 946 downregulated lncRNAs), 4101 mRNAs (770 upregulated and 3331 downregulated mRNAs; $\mid \log 2$ (fold_change) $\mid>1, q<0.001$ ), and 102 upregulated miRNAs (39 upregulated and 63 downregulated miRNA$\mathrm{s}$; $\mid \log 2$ (fold_change) $\mid>1, q<0.05)$. Among which, those with multiples of more than fivefold include 47 lncRNAs (maximum upregulation multiple of 38.11160073 and maximum downregulation multiple of 66.77770783), 47 miRNAs (maximum upregulation multiple of 142.82 and maximum downregulation multiple of 153.11), and 306 mRNAs (maximum upscaling multiple of 6700.53 and maximum downregulation multiple of 69.07). Of the differentially expressed lncRNAs, 184 belong to lincRNAs (16.93\%) and 220 belong to antisense lncRNAs (20.24\%). We also discovered 5029 novel lncRNAs by annotation; of which, 132 had significant differences (77 upregulated and 55 downregulated lncRNAs). The expression levels of miR-142-3p (downregulated) and miR-31-5p (downregulated) are consistent with those reported in previous studies. Among lncRNAs, the GAS5 expression decreased in the sequencing results (fold change down = 2.075, $q=5.89 \times 10^{-80}$ ). The lncRNA NEAT1 was significantly downregulated in patients with SLE, with a downregulation multiple of 1.498 and $q=8.882 \times 10^{-16}$. Furthermore, qPCR results showed that the expression of seven lincRNAs and two novel lncRNAs was consistent with the sequencing results; however, no significant difference was found in the expression of ENST00000542819.1. These results indicate the reliability of the sequencing results. We also found some of the differentially significant miRNAs in other diseases; these miRNAs include miR-135a-5p (up = 57.46) [37], miR-138-5p (down = 33.25) [38], miR-370-5p $($ down $=71.00)$ [39], and miR-490-3p (up = 7.26) [40], which may participate in the pathogenesis of SLE. The abovementioned lncRNAs and miRNAs with significant differences may serve as clinic markers for the diagnosis and evaluation of SLE activities and therapeutic effects.

To further clarify the mechanism of lncRNA in SLE, we selected the most studied and functional lincRNAs. Long intergenic noncoding RNAs (lincRNA) are a type of IncRNA that is transcribed from the gap between 
adjacent protein-coding genes. In lincRNAs, no overlap exists between the genomic region and the coding gene, and no coding gene exists within $30 \mathrm{~kb}$ in upstream and downstream $[41,42]$. The human body comprises thousands of lincRNAs, but only the functions of less than $1 \%$ have been identified [43]. SLE is a complex multi-gene disease. The completion of the Human Genome Project indicates that multiple loci are associated with SLE, and the genetic linkage has been confirmed with obvious correlations including 1q23-24, 1q31-32, 2p35-37, 4p16-15.2, 6p21-11, and 16q12-13 [44]. Therefore, we focused on lincRNAs located at the loci of SLE susceptibility and screened 30 lincRNAs combined with the expression quantity (RPKM) and multiples of diversity. For example, ENST00000524824.1, located at 11p15.5, where IRF7 is also located, may participate in the TLR/IFN signaling pathway [45]. ENST00000 601116.1 and ENST00000556030.1 are located in $19 q 13.2$ [46] and 15q26.2 [44], respectively, which are considered to be lupus gene susceptibility loci. ENST000 00417522.1 (1q32.1) is associated with IL10 and involved in the B cell signaling pathway [15]. ENST00000577528.1 (1p36.11) is associated with IL28RA and involved in the T cell signaling pathway [47].

Many lincRNAs coexpress with nearby coding genes and play an important role in transcriptional activation and phylogeny [48]. In this study, we constructed a lincRNA-mRNA coexpression network to discover the relationship between differential lincRNAs and differential mRNAs. We found that 23 lincRNAs and 353 mRNAs in 30 lincRNAs were involved in the network formation, forming 3782 lncRNA-mRNA expression pairs; of which, ENST00000339092.2 can be coexpressed with up to 284 mRNAs. The function of lncRNAs can be mainly detected by analysis of the effect of its target gene. Many lncRNAs can affect the expression of genes encoded within their vicinity in a cis-acting manner or regulate the translation of genes by complementary base pairing [12]. Therefore, we predicted the above 30 lincRNA target genes. The results show that 23 lincRNAs may act on 704 differential target genes, forming 890 lncRNA-gene connections (Additional file 4: Figure S1), except ENST00000596960.1; 7 successfully validated lincRNAs have more likely target genes, of which ENST00000601116.1 have the most 270 target genes. In the qPCR results, the most significantly upregulated and downregulated ENST00000524824.1 and ENST000005 77528.1 have 134 and 60 target genes, respectively. To further explore the biological functions of 23 lincRNAs, we conducted GO and pathway analyses on 740 differential target genes (Fig. 4). These genes were mainly enriched in the metabolic pathways, p53 signaling pathway, glycosphingolipid biosynthesis-globo series pathway, and FoxO signaling pathway, which are related to the pathogenesis of SLE. The metabolic abnormalities of lipids, proteins, and androgens in SLE have been widely reported. P53 is involved in the apoptosis and pathogenesis of SLE, and its expression level is significantly associated with SLE Disease Activity Index and the levels of anti-DNA antibody and IL-10 [19]. In glycosphingolipid biosynthesis-globo series, glycolipids and their derivatives play an important regulatory role in cell growth, differentiation, and apoptosis. Evidence also indicates that glycolipid molecules participate in the immune response process [20]. In the FoxO signaling pathway, FOX01 is negatively correlated with the activity of SLE [21]; the activation of FOX01 can stimulate the expression of Bim, which is a member of the pro-apoptotic $\mathrm{Bcl}-2$ gene family and plays an important role in apoptosis. We found that 62 coexpressed mRNAs are involved in the KEGG pathway changes and that the lincRNA-mRNA coexpression network is involved in the above pathways (Additional file 5: Figure S2, Additional file 6: Figure S3, Additional file 7: Figure S4). For example, 23 differential lincRNAs with 23 differential mRNAs are involved in metabolic pathways. In the p53 signaling pathway, 3 mRNAs interact with 17 lincRNAs. In the FoxO signaling pathway, 11 lincRNAs bind to 3 mRNAs. Based on the combined results of lincRNA-mRNA coexpression analysis and target gene prediction, 36 mRNAs were coexpressed with 1 of the 7 successfully validated lincRNAs and were predicted to be the corresponding lincRNA target genes. Forty-four pairs of lincRNA-mRNA were constructed. Some of the 36 mRNAs were confirmed to be one of the key genes in the pathogenesis of SLE. Interferon-induced protein with tetratricopeptide repeats 3 (IFIT3) is one of the genes which contribute to the overactive cGAS-STING signaling pathway in SLE monocytes, which may serve as a therapeutic target to block the production of type I IFN and other pro-inflammatory cytokines by cGAS-STING signaling pathway [49]. IFITM3 is a family of interferon-inducible transmembrane proteins (IFITM) that play a role in several biological activities, such as interferon homotypic cell adhesion and cell antiproliferative activity [50]. The expression level of IFITM3 was significantly higher in patients with SLE and was significantly negatively correlated with complement $\mathrm{C} 3$ and $\mathrm{C} 4$, which may be involved in the pathological process of SLE. IFIT3 (up = 3.65) and IFITM3 (up = 5.09) are the target genes of 3 lincRNAs (ENST00000524824.1, ENST00000531076.1, and ENST00000534483.1) and are coexpressed with them. Hence, these three lincRNAs may play an important role in the pathogenesis of SLE and affect disease activity by participating in the interferon (IFN) family and their immune regulatory pathways.

SLE in patients exhibits a strong gender bias. Was the differential expression of lncRNAs in our screen 
gender-dependent? TSIX, known as ENST0000060 4411.1 or LINC00013, expresses a noncoding antisense transcript across the $3^{\prime}$ end of the XIST locus. It also protects the active- $\mathrm{X}$ from ectopic silencing once $\mathrm{X}$-inactivation has commenced [23]. There is an increased incidence and prevalence of systemic lupus erythematosus in females, which might involve $\mathrm{X}$ chromosome inactivation [22]. In our study, we found that not only the expression of TSIX in SLE patients was significantly higher than that in healthy donors, but also the expression of TSIX in SLE female patients was significantly higher than that in SLE male patients. Therefore, the upregulated TSIX might facilitate X chromosome inactivation through protecting the active-X from ectopic silencing and take part in the pathogenesis of SLE.

Whether SLE patients exhibited a robust or weak "IFN signature"? Further, whether a particular SLEDAI score associated with the "IFN signature" or not? In recent years, research on the imbalance of cytokine networks in SLE has become increasingly deep, while IFN- $\alpha$ is an important regulator of the body's inflammation and immune response. In our study, the IFN- $\alpha$ level in the serum of SLE patients was higher than that in healthy donors, and the change was positively correlated with the SLE Disease Activity Index (SLEDAI), suggesting that IFN- $\alpha$ may play an important role in the SLE pathogenesis because it can activate autoreactive $\mathrm{T}$ and $\mathrm{B}$ lymphocytes and induce $B$ cells to produce antibodies. In addition, IFN- $\alpha$ can also induce the maturation and division of dendritic cells (DCs) and enhance the ability of DCs in capturing and presenting autoantigens, thereby breaking tolerance and accelerating the occurrence and development of SLE [51].

Whether PBMCs from SLE patients (as compared with healthy donors) exhibited differential expression of lncRNAs due to a change in the number of a cell type (e.g., lymphocytes, monocytes, and DCs) in peripheral blood? In most SLE patients, the expression of type-I IFN regulatory genes, also known as IFN signal, increased [52]. Some target genes of differentially expressed lncRNA in moDCs were associated with the type-I IFN system, such as IRF5. IRF5 is a target gene of lncRNA NR_034053.2 and is associated with an increase in IFN activity in the blood of SLE patients [53]. The increase in expression level of IncRNA NR_034053.2 in the moDCs of SLE patients may suggest that moDCs play a regulatory role in the production of lupus type I IFN [54]. Our results showed that, for PBMC and DCs isolated from the same volume of the whole blood of SLE patients and healthy donors, the expression levels of lncRNA NR_034053.2 in SLE patients were significantly higher than those in healthy donors, and for the T cells, $B$ cells, and monocytes isolated from the same volume of the whole blood of SLE patients, the expression level of lncRNA NR_034053.2 in monocytes was also significantly higher than that in $\mathrm{T}$ cells and $\mathrm{B}$ cells. These results might suggest PBMCs from SLE patients (as compared with healthy donors) exhibited differential expression of lncRNAs due to a change in the number of a cell type (e.g., lymphocytes, monocytes, and DCs) in the peripheral blood.

In 2011, Salmena et al. proposed competing endogenous RNA (ceRNA) theory, which reveals a new mechanism of RNA interaction [55]. ceRNAs can competitively bind to miRNAs through the same microRNA response elements (MREs), thereby affecting gene silencing caused by miRNA binding to mRNA to regulate gene expression [56]. In recent years, the function of lncRNAs as ceRNA-regulated gene expression has been elucidated in many diseases. Linc-MD1 binds to miR-133 and miR-135 through complementary base pairing and competitively inhibits their binding to target genes, thereby regulating muscle differentiation [57]. In colorectal cancer, IncRNA UICLM is a competitive endogenous RNA (ceRNA) that binds to miR-215 and suppresses the inhibition of ZIR2 by miR-215, thereby playing a role in promoting liver metastasis of colon cancer [58]. In view of the discovered lncRNAs, miRNAs, and other ncRNAs can participate in the biological processes of SLE occurrence and development. We can also infer the important relationship between ceRNA network imbalance and SLE disease. Through prediction of bioinformatics software, we constructed a ceRNA regulatory network among differential lncRNAs, miRNAs, and mRNAs. The ceRNA network contained 7 successfully validated significantly differential lincRNAs (3 upregulated and 4 downregulated), 15 significantly differential miRNAs (3 upregulated and 12 downregulated), and 155 significantly differential mRNAs (57 upregulated and 98 downregulated), forming 304 pairs of lncRNA-miRNA-mRNA. TCONS_00195779 (up = 4.39) can bind up to 10 miRNAs and form 64 lncRNA-miRNA-mRNA connections. miR-31-5p was found to be underexpressed in $T$ cells of patients with SLE; this molecule promotes the inhibition of the target gene RhoA and the downregulation of IL-2 expression and thus participates in immune disorders of SLE [24]. We can speculate that TCONS_00195779miR-31-5p-RhoA may be involved in the pathogenesis of lupus. In addition, miR-1268a ( down $=2.95)$, miR-143-3p (up = 2.07), and miR-182-5p (up = 2.13) can bind to 2, 3, and 2 lncRNAs, respectively, and could be the key nodes in the ceRNA network. In the target genes, we also found some susceptibility genes with SLE. IFIT1 and the abovementioned IFIT3 are the representative genes of the type I interferon system. The upregulated IFIT1 in lupus plays an important role in the production of cytokines IL- 4 and IL-10, which may be related to the imbalance of Th1/Th2. CXCL10, which belongs to the chemokine family, is 
significantly upregulated in SLE serum. CXCL10 can induce the chemotactic migration of macrophages, neutrophils, lymphocytes, and monocytes and mediate the aggregation and activation of leukocytes at the site of inflammation [59]. MALT1, a regulatory protein in the NF- $k \beta$ signaling pathway, plays an important role in the activation of NF- $\mathrm{k} \beta$ mediated by $\mathrm{T}$ cell receptor (TCR) and the regulation of lymphocyte proliferation and differentiation [60]. This protein was found to be significantly underexpressed in the PBMCs of patients with SLE [61], consistent with our sequencing results. Hence, we speculate that TCONS_00195779-miR-92a-1-5p-IFIT1 (up-down-up), TCONS_00195779-miR-3667-3p-CXCL10 (up-down-up), and ENST00000566788.1-miR-182-5pMALT1 (down-up-down) are a possible SLE-related lncRNA-miRNA-mRNA axis.

In summary, research on lncRNA in SLE has made some progress but is still in its infancy stage; related studies have focused on the discovery of differential lncRNAs.

\section{Conclusions}

In this study, we use full high-throughput sequencing to analyze the differences in the expression profiles of lncRNAs, miRNAs, and mRNAs in the PBMCs of patients with SLE at the genome-wide level. By using the gene loci and gene information of differentially expressed lncRNAs and bioinformatics analysis methods, we can select lincRNAs that may be closely related to SLE and preliminary ascertain their possible functions. We also further made experiments to confirm the following: (1) the differential expression of IncRNAs may be gender-dependent in SLE patients, (2) a positive correlation between IFN- $\alpha$ and disease activity in SLE patients, and (3) PBMCs exhibiting differential expression of lncRNAs may be due to a change in the number of a cell type. The results provide a foundation for clarifying the mechanism of IncRNAs in the pathogenesis of SLE and impart new ideas for effective treatment of SLE.

\section{Additional files}

Additional file 1: Table S1. Primer sequence for q-PCR. (DOCX $14 \mathrm{~kb}$ )

Additional file 2: Table S2. Fifteen significantly upregulated lincRNAs in SLE. (DOCX $12 \mathrm{~kb}$ )

Additional file 3: Table S3. Fifteen significantly downregulated lincRNAs in SLE. (DOCX $12 \mathrm{~kb})$

Additional file 4: Figure S1. Target gene prediction network map of 23 lincRNAs from 18 genes. (TIF $5716 \mathrm{~kb}$ )

Additional file 5: Figure S2. Twenty-three lincRNAs interacted with 23 mRNAs in the Metabolic pathways. (TIF $2422 \mathrm{~kb}$ )

Additional file 6: Figure S3. Seventeen lincRNAs interacted with 3 mRNAs in the meaningful p53 signaling pathway. (TIF $2190 \mathrm{~kb}$ )

Additional file 7: Figure S4. Eleven lincRNAs interacted with 3 mRNAs in the meaningful FoxO signaling pathway. (TIF $1765 \mathrm{~kb}$ )
Additional file 8: Figure S5. The mapping network of IncRNA-miRNA interactions between 9 dysregulated IncRNAs and 41 dysregulated miRNAs. (TIF $2488 \mathrm{~kb}$ )

Additional file 9: Figure S6. The cluster heat maps of 40 screened miRNAs:40 miRNAs were selected based on expression level and sequenced the multiple of the 102 differential miRNAs for the cluster analysis. (TIF $2567 \mathrm{~kb}$ )

Additional file 10: Figure S7. The mapping network of IncRNA-miRNAmRNA interactions between 4 downregulated IncRNAs, 3 upregulated miRNAs and 98 downregulated mRNAs. (TIF $2445 \mathrm{~kb}$ )

\section{Abbreviations}

CAMs: Cell adhesion molecules; ceRNA: Competing endogenous RNA; CPC: Coding Potential Calculator; DCs: Dendritic cells; ELISA: Enzyme-linked immunosorbent assay; GO: Gene Ontology; IFIT3: Interferon-induced protein with tetratricopeptide repeats 3; IFITM: Interferon-inducible transmembrane proteins; KEGG: Kyoto Encyclopedia of Genes and Genomes; Linc RNAs: Long intergenic noncoding RNAs; Lnc RNAs: Long noncoding RNAs;

miRNAs: MicroRNAs; MREs: MicroRNA response elements; PBMCs: Peripheral blood mononuclear cells; qRT-PCR: Real-time quantitative reverse transcriptase polymerase chain reaction; RT: Reverse transcription; SLE: Systemic lupus erythematosus; SLEDAl: Systemic Lupus Erythematosus Disease Activity Index; TCR: T cell receptor

\section{Acknowledgements}

We would like to thank the ShineWrite.com (Shanghai, China) for the helpful support in correcting the grammar of this paper.

\section{Funding}

This work was supported by grants from the Natural Science Foundation of Zhejiang Province, PRC (No. LY17H060008), the Zhejiang Provincial Foundation for Health Department (No.2015KYA150), the Wenzhou Municipal Science and Technology Project for Public Welfare (No.Y20140662), the National Natural Science Foundation of China (Grant No. 81472055 and 81672129).

\section{Availability of data and materials}

The datasets generated during and/or analyzed during the current study are available from the corresponding author on reasonable request.

\section{Authors' contributions}

All authors were involved in drafting the article or revising it critically for important intellectual content and approved the final version to be published. HY had full access to all of the data in the study and took responsibility for the integrity of the data and the accuracy of the data analysis. HY LW, and JW contributed to the study conception and design. HY, LW. XW, XC, and HZ contributed to the acquisition of data. LW, HY, ZW, XH, LS, $M J, H W$, and $X Y$ contributed to the analysis and interpretation of data.

\section{Ethics approval and consent to participate}

This study was approved by the Research Ethics Committee of Wenzhou Medical University (No. 2018925) and obtained informed consents from all participants.

\section{Consent for publication}

Written informed consents were obtained from the patients for publication of their individual details and accompanying images in this manuscript. The consent form is held by the authors and is available for review by the Editor-in-Chief.

\section{Competing interests}

The authors declare that they have no competing interests.

\section{Publisher's Note}

Springer Nature remains neutral with regard to jurisdictional claims in published maps and institutional affiliations.

\section{Author details}

${ }^{1}$ Department of Biochemistry, School of Basic Medical Sciences, Wenzhou Medical University, Wenzhou 325035, Zhejiang, China. ${ }^{2}$ School of the Second Clinical Medical Sciences, Wenzhou Medical University, Wenzhou 325035, 
Zhejiang, China. ${ }^{3}$ The First Affiliated Hospital of Wenzhou Medical University, Wenzhou 325035, Zhejiang, China. ${ }^{4}$ The Second Affiliated Hospital of Wenzhou Medical University, Wenzhou 325035, Zhejiang, China. ${ }^{5}$ School of Stomatology, Wenzhou Medical University, Wenzhou 325035, Zhejiang, China. ${ }^{6}$ Department of Medicinal Chemistry, School of Pharmaceutical Sciences, Wenzhou Medical University, Wenzhou 325035, Zhejiang, China.

\section{Received: 18 November 2018 Accepted: 24 February 2019}

\section{Published online: 05 March 2019}

\section{References}

1. Perl A. Pathogenic mechanisms in systemic lupus erythematosus. Autoimmunity. 2010;43:1-6.

2. Wu G, Jia X, Gao D, Zhao Z. Survival rates and risk factors for mortality in systemic lupus erythematosus patients in a Chinese center. Clin Rheumatol. 2014:33:947-53.

3. Ponting CP, Oliver PL, Reik W. Evolution and functions of Long noncoding RNAs. Cell. 2009;136:629-41.

4. Okazaki Y, Furuno M, Kasukawa T, Adachi J, Bono H, Kondo S, et al. Analysis of the mouse transcriptome based on functional annotation of 60,770 fulllength cDNAs. Nature. 2002;420:563-73.

5. Pagano A, Castelnuovo M, Tortelli F, Ferrari R, Dieci G, Cancedda R. New small nuclear RNA gene-like transcriptional units as sources of regulatory transcripts. PLoS Genet. 2007;3(2):e1.

6. Zhang K, Shi ZM, Chang YN, Hu ZM, Qi HX, Hong W. The ways of action of long non-coding RNAs in cytoplasm and nucleus. Gene. 2014;547:1-9.

7. Johnsson P, Lipovich L, Grandér D, Morris KV. Evolutionary conservation of long non-coding RNAs; sequence, structure, function. Biochim Biophys Acta. 2014;1840:1063-71.

8. Wu GC, Pan HF, Leng RX, Wang DG, Li XP, Li XM, et al. Emerging role of long noncoding RNAs in autoimmune diseases. Autoimmun Rev. 2015;14: 798-805.

9. Wu Y, Zhang F, Ma J, Zhang X, Wu L, Qu B, et al. Association of large intergenic noncoding RNA expression with disease activity and organ damage in systemic lupus erythematosus. Arthritis Res Ther. 2015;17:1-11.

10. Luo Q, Li X, Xu C, Zeng L, Ye J, Guo Y, et al. Integrative analysis of long non-coding RNAs and messenger RNA expression profiles in systemic lupus erythematosus. Mol Med Rep. 2018;17:3489-96.

11. Bombardier C, Gladman DD, Urowitz MB, Caron D, Chang CH. Derivation of the SLEDAl. A disease activity index for lupus patients. Arthritis Rheum. 1992:35:630-40

12. Guttman M, Rinn JL. Modular regulatory principles of large non-coding RNAs. Nature. 2012:482:339-46

13. Beissbarth T, Speed TP. GOstat: find statistically overrepresented Gene Ontologies within a group of genes. Bioinformatics. 2004:20:1464-5.

14. Huang DW, Sherman BT, Lempicki RA. Systematic and integrative analysis of large gene lists using DAVID bioinformatics resources. Nat Protoc. 2009;4: 44-57.

15. Gateva V, Sandling JK, Hom G, Taylor KE, Chung SA, Sun X, et al. A largescale replication study identifies TNIP1, PRDM1, JAZF1, UHRF1BP1 and IL10 as risk loci for systemic lupus erythematosus. Nat Genet. 2009;41:1228-33.

16. Zhao F, Qu Y, Liu J, Liu H, Zhang L, Feng Y, et al. Microarray profiling and co-expression network analysis of LncRNAs and mRNAs in neonatal rats following hypoxic-ischemic brain damage. Sci Rep. 2015;5:13850.

17. Dong R, Du J, Wang L, Wang J, Ding G, Wang S, et al. Comparison of long noncoding RNA and mRNA expression profiles in mesenchymal stem cells derived from human periodontal ligament and bone marrow. Biomed Res Int. 2014;2014:317853.

18. Yoon JH, Abdelmohsen K, Gorospe M. Post-transcriptional gene regulation by long noncoding RNA. J Mol Biol. 2013;425:3723-30.

19. Herkel J, Mimran A, Erez N, Kam N, Lohse AW, Märker-Hermann E, et al. Autoimmunity to the p53 protein is a feature of systemic lupus erythematosus (SLE) related to anti-DNA antibodies. J Autoimmun. 2001;17:63-9.

20. Zhou D. The immunological function of iGb3. Curr Protein Pept Sci. 2006;7: $325-33$

21. Kuo CC, Lin SC. Altered FOXO1 transcript levels in peripheral blood mononuclear cells of systemic lupus erythematosus and rheumatoid arthritis patients. Mol Med. 2007;13:561-6.

22. McCombe PA, Greer JM, Mackay IR. Sexual dimorphism in autoimmune disease. Curr Mol Med. 2009;9(9):1058-79.
23. Gayen S, Maclary E, Buttigieg E, Hinten M, Kalantry S. A primary role for the Tsix IncRNA in maintaining random X-chromosome inactivation. Cell Rep. 2015;11(8):1251-65.

24. Amr KS, Bayoumi FS, Elgengehy FT, Abdallah SO, Ahmed HH, Eissa E. The role of microRNA-31 and microRNA-21 as regulatory biomarkers in the activation of T lymphocytes of Egyptian lupus patients. Rheumatol Int. 2016;36:1-9.

25. Lerang K, Gilboe IM, Steinar Thelle D, Gran JT. Mortality and years of potential life loss in systemic lupus erythematosus: a population-based cohort study. Lupus. 2014;23:1546-52.

26. Wright MW, Bruford EA. Naming 'junk': human non-protein coding RNA (ncRNA) gene nomenclature. Hum Genomics. 2011;5:90-8.

27. Dai Y, Huang YS, Tang M, Lv TY, Hu CX, Tan YH, et al. Microarray analysis of microRNA expression in peripheral blood cells of systemic lupus erythematosus patients. Lupus. 2007;16:939.

28. Wang G, Tam LS, Li EK, Kwan BC, Chow KM, Luk CC, et al. Serum and urinary cell-free MiR-146a and MiR-155 in patients with systemic lupus erythematosus. J Rheumatol. 2010;37:2516-22.

29. Ding S, Liang $Y$, Zhao M, Liang G, Long $H$, Zhao S, et al. Decreased microRNA-142-3p/5p expression causes CD4+ T cell activation and B cell hyperstimulation in systemic lupus erythematosus. Arthritis Rheum. 2014;64: 2953-63.

30. Mayama T, Marr AK, Kino T. Differential expression of glucocorticoid receptor noncoding RNA repressor Gas5 in autoimmune and inflammatory diseases. Horm Metab Res. 2016;48:550-7.

31. Mourtada-Maarabouni M, Williams GT. Growth arrest on inhibition of nonsense-mediated decay is mediated by noncoding RNA GAS5. Biomed Res Int. 2013;2013:358015.

32. Zhang F, Wu L, Qian J, Qu B, Xia S, La T, et al. Identification of the long noncoding RNA NEAT1 as a novel inflammatory regulator acting through MAPK pathway in human lupus. J Autoimmun. 2016;75:96-104.

33. Su W, Xie W, Shang Q, Su B. The long noncoding RNA MEG3 is downregulated and inversely associated with VEGF levels in osteoarthritis. Biomed Res Int. 2015;2015:356893. https://doi.org/10.1155/2015/356893. Epub 2015 May 21.

34. Song J, Kim D, Han J, Kim Y, Lee M, Jin EJ. PBMC and exosome-derived Hotair is a critical regulator and potent marker for rheumatoid arthritis. Clin Exp Med. 2015;15:121-6.

35. Sun $Q L$, Zhao $C P$, Wang $T Y$, Hao $X B$, Wang $X Y$, Zhang $X$, et al. Expression profile analysis of long non-coding RNA associated with vincristine resistance in colon cancer cells by next-generation sequencing. Gene. 2015; 572:79-86.

36. Huo X, Han S, Wu G, Latchoumanin O, Zhou G, Hebbard L, et al. Dysregulated long noncoding RNAs (IncRNAs) in hepatocellular carcinoma: implications for tumorigenesis, disease progression, and liver cancer stem cells. Mol Cancer. 2017;16:165.

37. Zhao J, Li X, Zou M, He J, Han Y, Wu D, et al. miR-135a inhibition protects A549 cells from LPS-induced apoptosis by targeting Bcl-2. Biochem Biophys Res Commun. 2014;452:951-7.

38. Sen A, Most P, Peppel K. Induction of microRNA-138 by pro-inflammatory cytokines causes endothelial cell dysfunction. FEBS Lett. 2014;588:906-14.

39. Chang KW, Chu TH, Gong NR, Chiang WF, Yang CC, Liu CJ, et al. miR-370 modulates insulin receptor substrate-1 expression and inhibits the tumor phenotypes of oral carcinoma. Oral Dis. 2013;19:611-9.

40. Gu H, Yang T, Fu S, Chen X, Guo L, Ni Y. MicroRNA-490-3p inhibits proliferation of A549 lung cancer cells by targeting CCND1. Biochem Biophys Res Commun. 2014;444:104-8.

41. Atkinson SR, Marguerat S, Bähler J. Exploring long non-coding RNAs through sequencing. Semin Cell Dev Biol. 2012;23:200-5.

42. Song X, Cao G, Jing L, Lin S, Wang X, Zhang J, et al. Analysing the relationship between IncRNA and protein-coding gene and the role of IncRNA as ceRNA in pulmonary fibrosis. J Cell Mol Med. 2014;18:991-1003.

43. Khalil AM, Guttman M, Huarte M, Garber M, Raj A, Rivea Morales D, et al. Many human large intergenic noncoding RNAs associate with chromatinmodifying complexes and affect gene expression. Proc Natl Acad Sci U S A. 2009;106:11667-72.

44. Flesher DL, Sun X, Behrens TW, Graham RR, Criswell LA. Recent advances in the genetics of systemic lupus erythematosus. Expert Rev Clin Immunol. 2010;6:461-79.

45. Chung SA, Taylor KE, Graham RR, Nititham J, Lee AT, Ortmann WA, et al. Differential genetic associations for systemic lupus erythematosus based on anti-dsDNA autoantibody production. PLoS Genet. 2011;7:e1001323. 
46. Cunninghame Graham DS, Morris DL, Bhangale TR, Criswell LA, Syvänen AC, Rönnblom L, et al. Association of, NCF2, IKZF1, IRF8, IFIH1, and, TYK2, with systemic lupus erythematosus. PLoS Genet. 2011;7:e1002341.

47. Li Y, Cheng H, Zuo XB, Sheng YJ, Zhou FS, Tang XF, et al. Association analyses identifying two common susceptibility loci shared by psoriasis and systemic lupus erythematosus in the Chinese Han population. J Med Genet. 2013;50:812-8

48. Guttman M, Amit I, Garber M, French C, Lin MF, Feldser D, et al. Chromatin signature reveals over a thousand highly conserved large non-coding RNAs in mammals. Nature. 2009;458:223-7.

49. Wang J, Dai M, Cui Y, Hou G, Deng J, Gao X, et al. Elevated IFIT3 contributes to abnormal overactive CGAS-STING signaling in human systemic lupus erythematosus monocytes. Arthritis Rheumatol. 2018;70:2036-45.

50. Bailey CC, Kondur HR, Huang IC, Farzan M. Interferon-induced transmembrane protein 3 is a type II transmembrane protein. J Biol Chem. 2013;288:32184-93.

51. Blanco P, Palucku AK, Gill M. Induction of dendritic cell differentiation by IFN-alpal in systemic lupus erythematosus. Science. 2001;294:1540-3.

52. Bengtsson AA, Ronnblom L. Role of interferons in SLE. Best Pract Res Clin Rheumatol. 2017;31(3):415-28.

53. Niewold TB, Kelly JA, Flesch MH, Espinoza LR, Harley JB, Crow MK. Association of the IRF5 risk haplotype with high serum interferon-alpha activity in systemic lupus erythematosus patients. Arthritis Rheum. 2008; 58(8):2481-7.

54. Wang Y, Chen S, Chen S, Du J, Lin J, Qin H, Wang J, Liang J, Xu J. Long noncoding RNA expression profile and association with SLEDAl score in monocyte-derived dendritic cells from patients with systematic lupus erythematosus. Arthritis Res Ther. 2018;20:138-48.

55. Salmena L, Poliseno L, Tay Y, Kats L, Pandolfi PP. A ceRNA hypothesis: the Rosetta stone of a hidden RNA language? Cell. 2011;146:353-8.

56. Bartel DP. MicroRNAs: target recognition and regulatory functions. Cell. 2009;136:215-33.

57. Legnini I, Morlando M, Mangiavacchi A, Fatica A, Bozzoni I. A feedforward regulatory loop between HuR and the long noncoding RNA linc-MD1 controls early phases of myogenesis. Mol Cell. 2014;53:506-14.

58. Chen DL, Lu YX, Zhang JX, Wei XL, Wang F, Zeng ZL, et al. Long noncoding RNA UICLM promotes colorectal cancer liver metastasis by acting as a ceRNA for microRNA-215 to regulate ZEB2 expression. Theranostics. 2017; 7:4836-49.

59. Kong KO, Tan AW, Thong BY, Lian TY, Cheng YK, Teh CL, et al. Enhanced expression of interferon-inducible protein-10 correlates with disease activity and clinical manifestations in systemic lupus erythematosus. Clin Exp Immunol. 2009;156:134-40.

60. He Y, Xu Y, Zhang C, Gao X, Dykema KJ, Martin KR, et al. Identification of a lysosomal pathway that modulates glucocorticoid signaling and the inflammatory response. Sci Signal. 2011:4(180):ra44. https://doi.org/10.1126/ scisignal.2001450.

61. Li D, Wang L, Fan Y, Song L, Guo C, Zhu F, et al. Down-regulation of A20 mRNA expression in peripheral blood mononuclear cells from patients with systemic lupus erythematosus. J Clin Immunol. 2012;32:1287-91.

Ready to submit your research? Choose BMC and benefit from:

- fast, convenient online submission

- thorough peer review by experienced researchers in your field

- rapid publication on acceptance

- support for research data, including large and complex data types

- gold Open Access which fosters wider collaboration and increased citations

- maximum visibility for your research: over $100 \mathrm{M}$ website views per year

At BMC, research is always in progress.

Learn more biomedcentral.com/submissions 